\title{
Diseño integrado de seminarios y prácticas de laboratorio por medio de un proyecto de investigación
}

Antoni Sureda $^{a, b}$, Xavier Capóa ${ }^{a}$, Samuel Pinya ${ }^{c}$, Pere Ferriol ${ }^{c}$, Guillem Mateu-Vicens ${ }^{\mathrm{d}, \mathrm{e}}$, Silvia Tejada $^{\text {b,f }}$

\footnotetext{
${ }^{a}$ Research Group on Community Nutrition and Oxidative Stress (NUCOX) and IdISBa University of Balearic Islands, E-07122 Palma de Mallorca, Balearic Islands, Spain, ${ }^{\mathrm{b}}$ CIBEROBN (Physiopathology of Obesity and Nutrition), E07122 Palma de Mallorca, Balearic Islands, Spain, antoni.sureda@uib.es, ${ }^{c}$ Interdisciplinary Ecology Group, Biology Department, University of the Balearic Islands, E-07122 Palma de Mallorca, Spain, s.pinya@uib.es, pere.ferriol@uib.es, ${ }^{\mathrm{d}}$ Museu Balear de Ciències Naturals, Ctra. Palma - Port de Sóller km 30, 07100 Sóller, Balearic Islands, Spain, ${ }^{e}$ Laboratory of Zoology, Biology Department, University of the Balearic Islands, E-07122 Palma de Mallorca, Spain, guillem.mateu@uib.es, ${ }^{\mathrm{f}}$ Laboratory of Neurophysiology, Biology Department, University of the Balearic Islands, E-07122 Palma de Mallorca, Spain, silvia.tejada@uib.es.
}

\section{\$EWWFW}

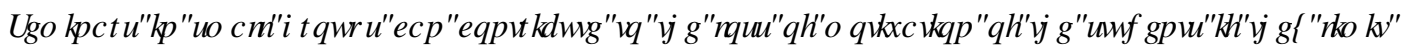

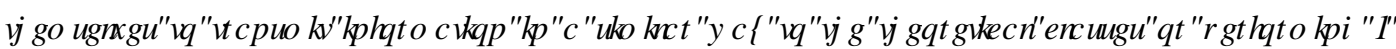

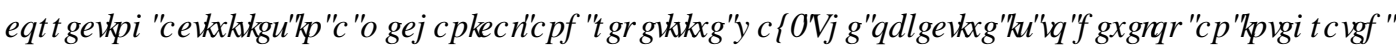

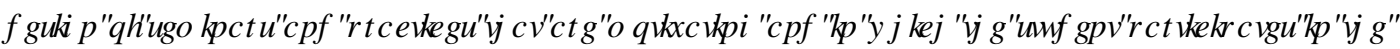

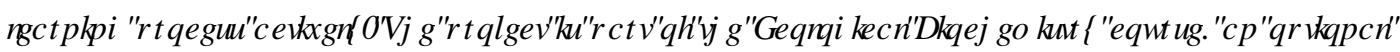

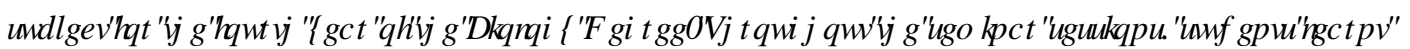

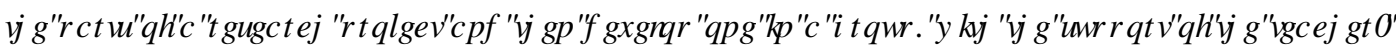

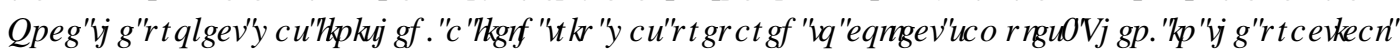

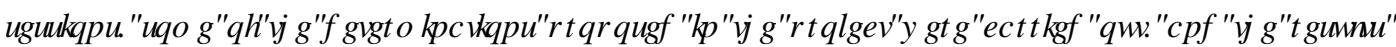

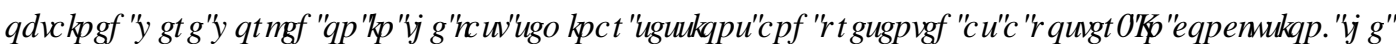

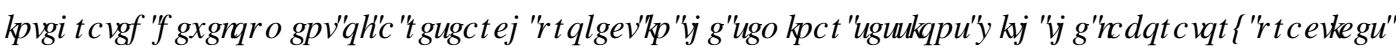

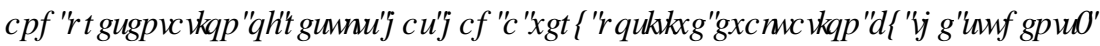

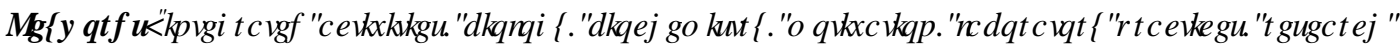
SLRNAFWUP LQDW

\section{HXP HQ}

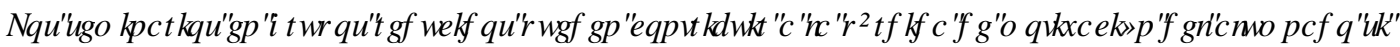

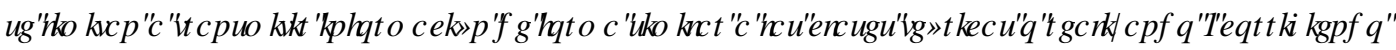

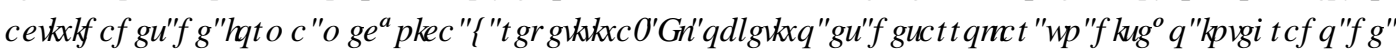

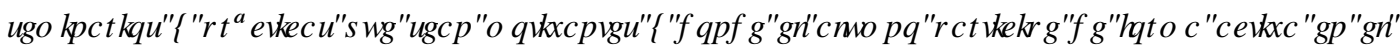

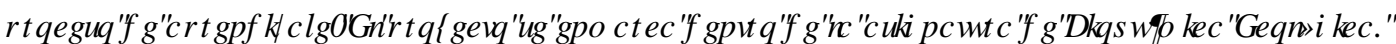

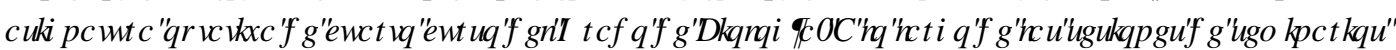

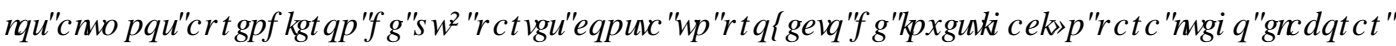

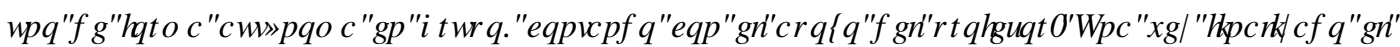

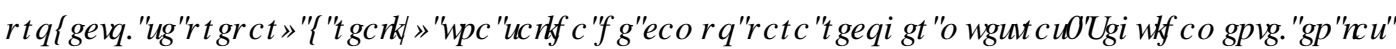

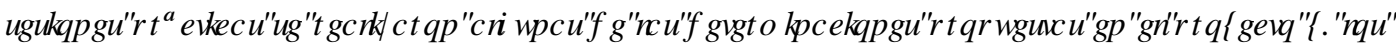

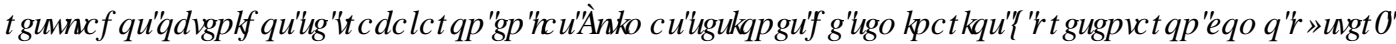

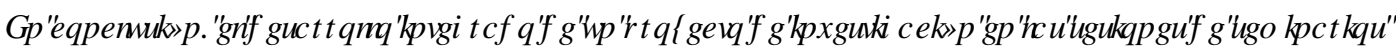




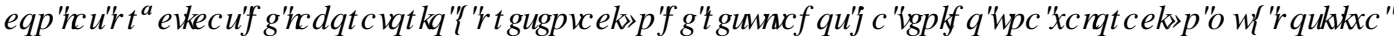 SRUSDUИAGHIRVHMUGDQUTL}

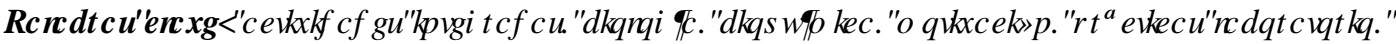 SUR HFURIQYHMU DFIY QUИP LQDURVD}

\section{Introducción}

De acuerdo a los criterios y exigencias derivados de la implantación del Espacio Europeo de Educación Superior (EEES) es esencial que la enseñanza superior universitaria se convierta en un entorno dinámico y comunicativo que fomente el interés y la curiosidad, en este caso científica, a los alumnos que eligen unos estudios de grado (1). Para favorecer que los alumnos muestren interés por las asignaturas es fundamental tener una visión global del contenido de las mismas y ofrecer al alumnado una coherencia en los contenidos y el desarrollo de los aspectos más prácticos que van a cursar. De esta manera se pueden llevar a cabo propuestas didácticas integradas y cooperativas, como una nueva forma de aprender y de enseñar, tanto en los conceptos que el alumnado debe adquirir como con las habilidades alcanzadas en las salidas de campo o las prácticas el laboratorio $(2,3)$.

Un aspecto importante de los estudios de grado es que implican una gran diversidad de metodologías docentes - clases, seminarios, trabajo interactivo en grupo, equipos de trabajo, trabajos en proyectos, laboratorios, ejercicios de campo, trabajos de ordenador, escritura de trabajos, etc. Referente al trabajo que se realiza en las sesiones de seminarios / talleres con grupos medianos, es muy habitual seguir trabajando de una forma magistral y la realización de ejercicios o problemas derivados de las clases teóricas. Todo ello contribuye a que el alumnado no sienta que se están realizando actividades distintas de las del grupo grande y contribuye a una desmotivación de los mismos. El método de aprendizaje mediante proyectos emerge de una visión de la educación en la que los estudiantes toman una mayor responsabilidad de su propio aprendizaje y donde se aplican, en proyectos reales, las habilidades y conocimientos adquiridos a lo largo de los estudios de grado (4). El uso de proyectos busca enfrentar a los alumnos a situaciones que los llevan a recordar, comprender y aplicar lo que aprenden como una herramienta para resolver problemas o proponer mejoras en las comunidades donde se desarrollan. Mediante el presente proyecto se pretende promover la participación y la motivación de los alumnos mediante un trabajo integrado donde las diferentes actividades planteadas en las sesiones de seminarios permitan su aplicación en las sesiones de prácticas. Todo ello permitirá que los alumnos puedan obtener unos resultados y unas conclusiones por sí mismos y sentirse partícipes del trabajo realizado.

\section{Objetivos}

El objetivo principal del presente proyecto es el de desarrollar un trabajo coordinado dentro de las sesiones de seminarios y de prácticas a lo largo del desarrollo de toda la asignatura de Bioquímica Ecológica del grado de Biología.

Para ello, se propone a los alumnos trabajar en la elaboración y redacción de un proyecto de investigación dentro del marco de la Bioquímica Ecológica desarrollando todos los apartados requeridos, y la realización de parte del procedimiento experimental dentro de las prácticas de la asignatura. Finalmente, se tratan estadísticamente los resultados obtenidos y se presentan y discuten en formato póster. 


\section{Desarrollo de la innovación}

El aprendizaje Basado en Proyectos en el que se basa el presente proyecto de innovación es una metodología de aprendizaje en la que los estudiantes adquieren un papel activo y se favorece la motivación académica. El método consiste en la realización de un proyecto habitualmente en grupo. Este proyecto será analizado previamente por el profesor para asegurarse de que el alumno tiene todo lo necesario para resolverlo, y que en su resolución desarrollará todas las destrezas que se desea. En este tipo de proyecto el alumnado puede participar, hablar y dar su opinión mientras que el profesorado adquiere un papel menos activo ayudando a conseguir un consenso y orientar el desarrollo del proyecto del alumnado.

Para alcanzar el objetivo de la propuesta se procedió al desarrollo de forma integrada de un trabajo dentro de las sesiones en grupo medio que permitió a los alumnos conocer todos los aspectos que conforman un proyecto de investigación, desde su redacción, hasta la su realización y comunicación de los resultados. En la primera sesión de seminario se dedicaron los primeros minutos a responder un corto cuestionario inicial de cinco preguntas sobre proyectos de investigación y la posibilidad de trabajar de forma integrada a los alumnos para determinar sus conocimientos iniciales y a predisposición a la nueva metodología. De forma adicional, al final del cuestionario los alumnos tenían espacio para explicar de forma libre lo que creyeran conveniente. En el cuestionario se preguntaba lo siguiente:

- ¿Has elaborado en alguna ocasión un proyecto de investigación?

- ¿Conoces todas las secciones que forman parte de un proyecto de investigación?

- ¿Has presentado en alguna ocasión los resultados de práctica en forma de póster?

- ¿Crees interesante trabajar de forma integrada un proyecto de investigación y su desarrollo en la asignatura de Bioquímica Ecológica?

- ¿Puede ser importante para mi futuro saber elaborar un proyecto de investigación?

Una vez concluido el cuestionario, en las dos primeras sesiones de grupo medio se presentaron los diferentes apartados del que consta un proyecto de investigación mostrando diferentes ejemplos de casos reales. Entre los diferentes casos, se mostraron algunos con carencias para que los alumnos las encontraran y propusieran alternativas para poder solucionarlas.

A partir de aquí se comenzó a trabajar sobre un proyecto conjunto con el título "Efectos de la salinidad asociada al spray salino del mar sobre plantas mediterráneas mediante la evaluación de biomarcadores". En las siguientes sesiones los alumnos fueron desarrollando los diferentes apartados del proyecto (antecedentes, hipótesis, objetivos, procedimiento experimental, presupuesto, etc.) con la ayuda y asesoramiento del profesor. Una vez finalizada la parte de redacción, se procedió a coordinar entre alumnos y profesores la salida de campo (prevista dentro del cronograma de la asignatura) para recoger las muestras de material vegetal de forma adecuada. El día de la salida los alumnos recogieron el número necesario de muestras, utilizando las metodologías que garantizaban el correcto manejo y mantenimiento de las mismas hasta que lleguen de nuevo a la Universidad donde se mantuvieron congeladas.

Tras la salida de campo, la asignatura dedicó dos sesiones prácticas de laboratorio de 4 horas cada unapara el procesamiento y análisis de las muestras (se realizaron sólo una parte de las determinaciones previstas en el proyecto original por cuestiones de tiempo y económicas). En las últimas sesiones de grupo medio se trabajó la realización de los cálculos a partir de los datos obtenidos, su análisis estadístico y su presentación en formato póster.

Finalmente, se elaboraró un cuestionario (cada respuesta se valora entre 0 y 10 , y una pregunta final de respuesta libre) para que los alumnos la pudieran completar con el objetivo de conocer si el hecho de trabajar un proyecto de investigación de forma integrada los motiva y si creen que les podrá ser de utilidad a su próximo futuro profesional. Se dejó una pregunta de respuesta abierta para que los alumnos pudieran 
expresar su opinión y hacer propuestas de mejora. Mediante este cuestionario se pretendía que los alumnos valoraran las diferentes etapas asociadas al propio desarrollo del proyecto, desde la búsqueda de información inicial a la realización de cálculos y la presentación final de resultados en forma de póster. Además, se incorporaron preguntas más generales para que valoraran la carga de trabajo que les ha supuesto el desarrollo del proyecto, si la organización de la asignatura es atractiva y una valoración global final. Y como última pregunta, se trata el tema de la utilidad de todo lo realizado para su futuro profesional.

\section{Resultados}

La Bioquímica Ecológica es una asignatura optativa de cuarto curso, por lo que el número de alumnos es relativamente bajo facilitando el trabajar en aspectos de innovación docente. En el curso académico 201819 se matricularon en la asignatura un total de 12 alumnos, 5 provenientes del grado en Biología y 7 del grado en Bioquímica.

Las respuestas al cuestionario inicial se presentan en la siguiente tabla:

7DECDI T5 HXQDDRVGHFXHMRQDURIQLFIDO

\begin{tabular}{lcc}
\hline Pregunta & Sí & No \\
\hline ¿Has elaborado en alguna ocasión un proyecto de investigación? & 5 & 7 \\
¿Conoces todas las secciones que forman parte de un proyecto de investigación? & 3 & 9 \\
¿Has presentado en alguna ocasión los resultados de práctica en forma de póster? & 2 & 11 \\
$\begin{array}{l}\text { ¿Crees interesante trabajar de forma integrada un proyecto de investigación y su } \\
\text { desarrollo en la asignatura de Bioquímica Ecológica? }\end{array}$ & 12 & 0 \\
¿Puede ser importante para mi futuro saber elaborar un proyecto de investigación? & 10 & 2 \\
\hline
\end{tabular}

A la primera pregunta respondieron de forma afirmativa los 5 alumnos provenientes del grao en Biología porque tienen una asignatura específica de elaboración de proyectos científicos, aunque muy dirigidos a exponer los diferentes apartados de los proyectos a nivel nacional de financiación pública y no encaminados a un ámbito más aplicado y local. Sin embargo, únicamente 3 de los alumnos afirmaron acordarse de las diferentes secciones que forman parte de un proyecto de investigación, lo que hace importante el trabajo que se plantea en el presente proyecto. De todos los alumnos, sólo dos han elaborado en alguna ocasión una presentación en formato pósters, y se debe a que coincidieron en una asignatura optativa donde se les pidió presentar unos datos en este formato. A las preguntas sobre el interés de trabajar de forma integrada un proyecto, todos ellos indicaron que lo veían interesante, sobre todo porque se seguía un hilo de trabajo durante el curso, aunque dos de ellos pensaban que se realización no les serviría de mucho en su futuro. Hay que indicar que estos dos alumnos querían dedicarse a la docencia y no veían la necesidad de saber elaborar este tipo de proyectos. En el espacio destinado a la respuesta libre únicamente 8 de los almumnos hicieron aportaciones, mientras que los otros cuatro dejaron el espacio en blanco. Entre las respuestas se puede destacar que la mayoría comentaba que no tienen muy claro cómo debe elaborarse un proyecto lo que les despierta interés y curiosidad, y algunos de los que ya lo habían trabajado anteriormente comentaron que lo recuerdan como sesiones muy teóricas sin ver una aplicación clara. $\mathrm{Y}$ de forma general, los alumnos indican que para la carrera científica es esencial saber elaborar un proyecto para poder investigar y conseguir financiación.

Referente al desarrollo completo de un proyecto de investigación, los alumnos han podido aprender todo el proceso relacionado con un proyecto científico, desde su diseño y redacción, hasta llevarlo a cabo (en parte) y presentar los resultados con sus conclusiones en forma de póster. Como era de esperar, los principales problemas que han surgido a la hora de elaborar el proyecto se han centrado en la parte de metodología, ya 
que es donde los alumnos deben decidir qué quieren realmente analizar y con qué finalidad. También se han detectado dificultades en la elaboración de la hipótesis / objetivos, ya que les cuesta separar una idea de la otra. Por otro lado, el destinar tiempo a preparar las prácticas de laboratorio durante los seminarios ha permitido que tanto la salida de campo como las propias prácticas en el laboratorio se desarrollaran de forma ágil y sin ningún tipo de problema inesperado. Finalmente, los alumnos han agradecido el destinar una sesión final a la realización de los cálculos porque según ellos es una parte que se trabaja poco en las prácticas y siempre les genera dudas. Para la elaboración de un póster con los resultados propios, se facilitó a los alumnos algunos ejemplos propios para que pudieran usarlos de base para su propia realización. Así, los alumnos han presentado pósteres muy visuales y correctos que podrían ser presentados en algún congreso de investigación.

Los resultados obtenidos por medio del cuestionario contestado por los alumnos al final del curso se presentan en la Figura 1.

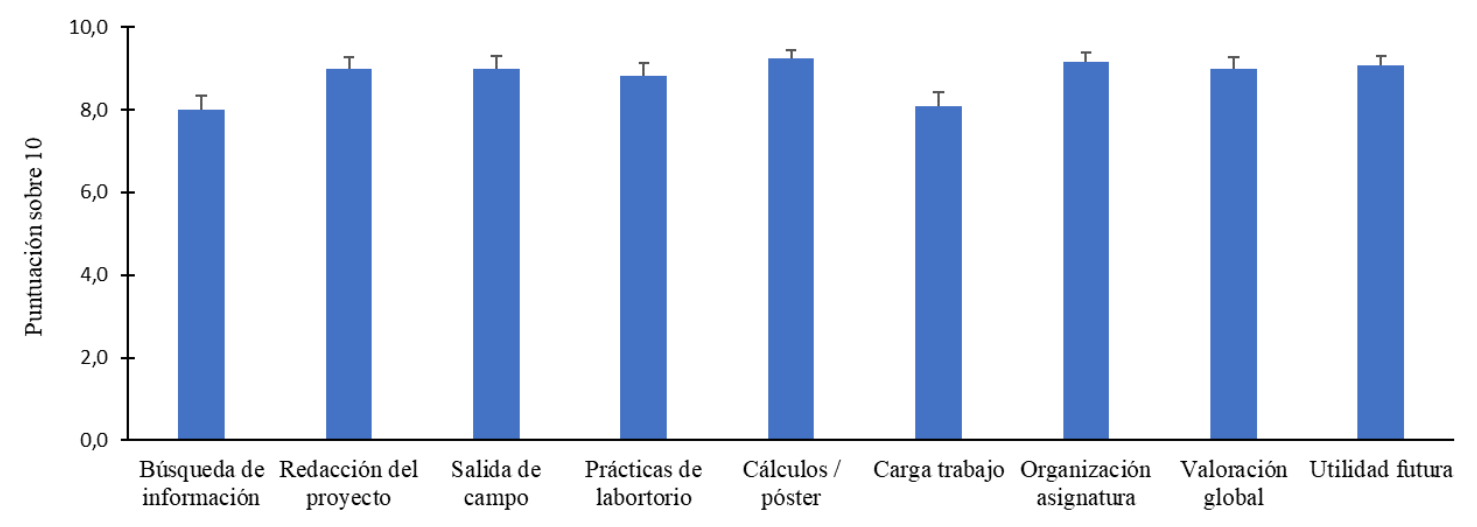

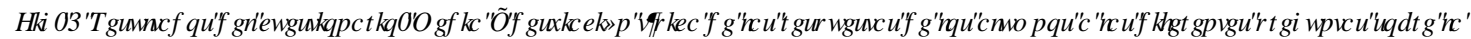

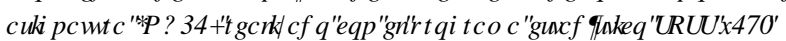

El cuestionario realizado pone en evidencia que los alumnos han quedado satisfechos con el desarrollo del proyecto, ya que todas las preguntas han obtenido al menos un 8 como nota media. Los dos aspectos que han obtenido la peor puntuación han sido el tiempo de trabajo que los alumnos han tenido que dedicar a la búsqueda de información para luego realizar el proyecto y la propia carga de trabajo de la redacción del proyecto de investigación. Tras tratarlo con los alumnos, éstos han indicado que el problema con la carga de trabajo deriva principalmente del hecho que con anterioridad no habían redactado ningún proyecto por ellos mismos y les ha costado mucho sobre todo la parte del procedimiento experimental. Por el contrario, lo que más ha gustado a los alumnos es el poder dedicar una sesión para realizar los cálculos con los resultados obtenidos y luego poder plasmarlos de forma visual en formato póster. Finalmente, indicar que la organización general de la asignatura, la valoración global de todo el proceso de elaboración de un proyecto y su posible utilidad futura han obtenido al menos un 9 de puntuación, lo que es indicativo del buen funcionamiento y motivación respecto al proyecto desarrollado. En este sentido, la buena acogida de la asignatura se reflejó con las calificaciones obtenidas por parte de los alumnos ya que todos ellos aprobaron la asignatura en la primera convocatoria con 4 de ellos con la calificación de excelente y un 7 con la de notable y un único aprobado. 


\section{Conclusiones}

A modo de conclusión, el presente proyecto ha permitido trabajar de forma integrada y coordinada un proyecto de investigación a lo largo de toda la asignatura de Bioquímica Ecológica. Gracias a este proyecto, los alumnos han podido sentirse partícipes de todo el proceso, desde la fase inicial para decidir el tipo de proyecto a elaborar hasta la presentación final de los resultados en forma de póster. Creemos que esta forma de trabajar contribuye a incrementar de forma significativa la motivación y la implicación de los alumnos en todos los aspectos del trabajo realizado. Entre los aspectos a mejorar sería intentar reducir la carga de trabajo que ha supuesto para los alumnos, sobre todo la parte de búsqueda de información y redacción del proyecto filtrándoles la bibliografía necesaria para el proyecto.

\section{Referencias}

EUROPEAN ASSOCIATION FOR QUALITY ASSURANCE IN HIGHER EDUCATION. (2005). "Criterios y Directrices para la Garantía de Calidad en el Espacio Europeo de Educación Superior". $<$ http://www.enqa.net/bologna.lasso $>$ [Consulta: 16 de mayo de 2018].

MORALES, P. (2007). "Nuevos roles de profesores y alumnos, nuevas formas de enseñar y de aprender".

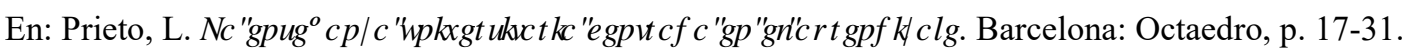

PÉREZ, M. M. (2008). “Aprendizaje basado en proyectos colaborativos. Una experiencia en educación superior" en / DXUXV, 14, 28, 158-180.

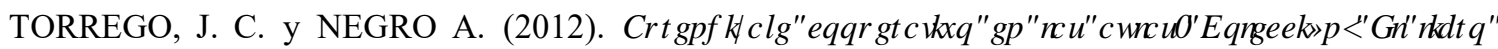
XQIYHULDURTO DQXDON. Madrid: Alianza Editorial.

YAVUZ ORUC, A. (2017). + DQCERRNIRIL6FIHQWIFL3 LRSRWD: UUQJ. Boca Raton: Editorial Chapman and Hall/CRC.

Agradecimientos: el proyecto ha sido financiado por la Universitat de les Illes Balears - Institut de Recerca i Innovació Educativa. 